\title{
Surface State Electrons on Solid Hydrogen Films
}

\author{
U.ALBRECHT, H.DILGER, P.LEIDERER, Fakultät für Physik, Universität Konstanz, D-7750 Konstanz, \\ West Germany $\dagger$ \\ K.KONO, Cryogenics Center, University of T'sukuba, 305 Japan $\ddagger$
}

We investigated the surface of quench condensed hydrogen films on a glass substrate by measuring the conductivity of surface state electrons (SSE) between 1.5 and $4.2 \mathrm{~K}$. Initially the films were very rough (no conductivity signal). After thermal annealing the SSE conductivity increased drastically, behaving with temperature like a thermally activated process. Saturated helium films on the hydrogen led to a large increase in the SSE conductivity. Shortly before helium film completion a sharp dip in the conductivity is observed.

\section{Introduction}

Solid hydrogen surfaces have become of considerable interest recently $[1,2,3]$ as a substrate for surface state electrons (SSE) alternative to liquid helium, where a particularly clean and well defined 2-dimensional electron system can be prepared. One might hope to achieve a high electron density on solid hydrogen films, which for liquid helium is limited to $u=2 \cdot 10^{9} \mathrm{~cm}^{-2}$ by the electrohydrodynamic instability of the surface [4]. This motivated us to investigate the surface of quench condensed hydrogen films, using the SSE as a tool to probe the surface quality by measuring their ac-conductivity.

\section{Experimental}

The hydrogen films were quench condensed onto a floatglass plate of $0.2 \mathrm{~mm}$ thickness at $1.5 \mathrm{~K}$, which was mounted onto two concentric copper electrodes. These were used to measure the ac-conductivity of the SSE by means of the Sommer-Tanner method [5]. We prepared films of about $2 \mu \mathrm{m}$ thickness (measured interferometrically). After preparation the films were charged with electrons from a tungsten filament. A small amount of helium gas inside the cell served to reduce the mean free path of the electrons in order to prevent them from penetrating the hydrogen film.

\section{Results}

After charging the surface the conductivity $\sigma$ due to the SSE was measured as a function of temperature, which was swept at about $2 \mathrm{~K} /$ hour. Figure 1 shows traces of $\sigma$ versus ' $T$ ' before and after annealing of the film. The high

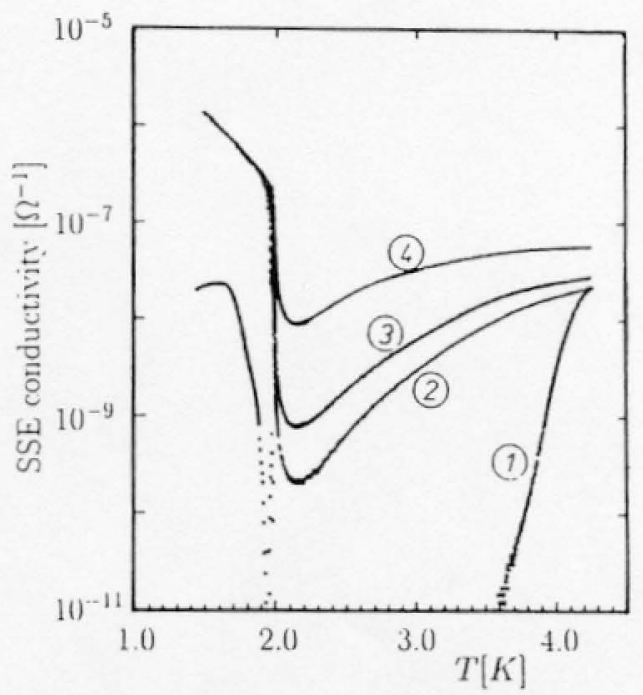

Figure 1: SSE conductivity on hydrogen film for various annealing steps (as described in the text)

mobility on the low temperature end of the scans is due to the helium in the cell, which forms a saturated film of about $30 \mathrm{~nm}$ thickness below a certain temperature $T_{0}$, depending on the amount of helium gas in the cell (in the case of fig. 1 roughly at $1.9 \mathrm{~K}$ ). The conductivity on such a thick $\mathrm{He}$ film is close to the value on bulk helium [2]. Raising the temperature above $T_{\mathrm{u}}$ leads to the disappearance of the $\mathrm{He}$ film into the gas phase, and we measure the conductivity of the bare hydrogen film. The SSE conductivity of the freshly prepared film ( $\operatorname{scan} 1$ ) is below our sensitivity threshold of $10^{-11} \Omega \Omega^{-1}$ up to about $3.5 \mathrm{~K}$, where it is seen to grow with temperature. This is mainly due to annealing effects as can be seen in scan 2 where $T$ is swept back down to $1.5 \mathrm{~K}$

$\dagger$ Work supported by the Deutsche Forschungsgeneinschaft (SFB 306)

thi. Kono acknowledges support by the Alexander von llumboldt-Stiftung, and the Japantese Ministry of biducation Science and Chalture 
right after reaching $4.2 \mathrm{~K}$. An increase in the signal of at least two orders of magnitude is observed. Scans 3 and 4 show the effects of further annealing (scan 3: 12 hours at $4.2 \mathrm{~K}$, scan 4: annealing up to $8 \mathrm{~K}$ ). Scans 2 to 4 were reversible on a scale of about an hour.

Fig. 2 shows the part of interest of these scans in an Arrhenius plot. Evidently the conductivity is governed by thermal activation. The activation energies $\Delta E$ decrease with progressive annealing, the lowest obtained being about $10 \mathrm{~K}$. Since the conductivity is given by $\sigma=\mu n_{e / f} e$ (where $e$ is the electron charge, $n_{e f f}$ is the fraction of the electrons which takes part in the transport process, and $\mu$ is the mobility of these electrons), the variation of $\sigma$ with temperature can be ascribed to a temperature dependence of $\mu$ and/or $n_{e j /}$ (conductivity measurements in a magnetic field will allow to distinguish between these possibilities). Assuming that the main contribution is due to neff, the data reflect the thermal activation of electrons from traps formed by surface irregularities. The observed decrease of $\Delta E$ on annealing then gives the reduction of the average trap energy.

There is practically no difference between the annealed scans in the helium film region (i.e. $T<T_{0}$ ). Obviously the surface structure of these films does not affect the surface of the saturated $\mathrm{He}$ film to an extent that would influence the SSE conductivity. During growth of the He film the SSE conductivity does not increase monotonically, but shows a sharp dip shortly before completion of the film, as shown in fig. 3. This has been observed before on other substrates and bulk hydrogen as well $[1,6]$. The nature of this effect, which has been attributed to enhanced ripplon scattering, is still speculative, though. We are currently setting up an experiment to determine accurately the film thickness. where the conductivity anomaly occurs.

\section{Conclusions}

We have measured the ac-conductivity of surface state electrons (SSE) on quench condensed hydrogen films. Right after preparation at $1.5 \mathrm{~K}$ the films were so rough, that the SSE conductivity was below our detection limit of $10^{-11} \Omega^{-1}$. Thermal annealing improved the surface quality drastically. The temperature dependence of the SSE conductivity showed the characteristics of a thermally activated process, the activation energy decreasing on annealing. Hydrogen films thus appear as a promising substrate for 2-dimensional electron systems at high densities.

Saturated helium films on the hydrogen greatly increased the SSE conductivity. We confirmed the appearance of a sharp dip in the conductivity shortly before completion of the $\mathrm{He}$ film, which had already been reported also on other substrates.

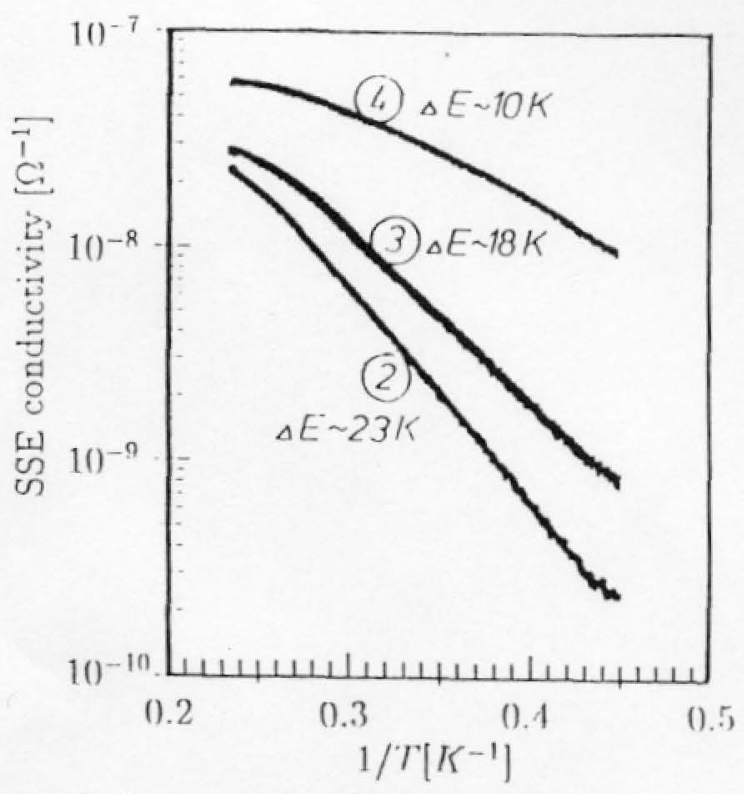

Figure 2: Arrhenius plot of scans 2-4 of figure 1

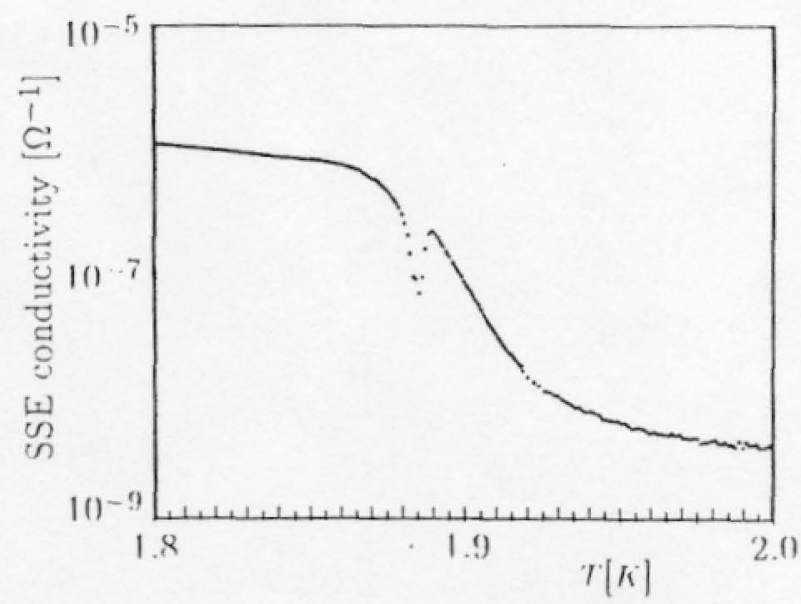

Figure 3: SSE conductivity during growth of helium film on hydrogen

\section{References}

[1] M.A.Paalanen and Y.lye. Phys.Rev.Lett.55 (1985) 1761

[2] D.Cieslikowski, P.Leiderer and A.J.Dahm, Can.J.Phys.65 (1987) 1525

[3] P.W.Adams and M.A.Paalanen, Phys.Rev.Lett.58 (1987) 2106

[4] P.Leiderer, Physica B+C(Amsterdam) 126 (1984) 126

[5] W.T.Sommer and D.J.Tanner, Phys.Rev.Lett.27 (1971) 1845

[6] E.Y.Andrei, Phys.Rev.Lett. 53 (1984) 1499 